\title{
Role of Wnt/ $\beta$-catenin pathway in inducing autophagy and apoptosis in multiple myeloma cells
}

\author{
NAN SU, PINGPING WANG and YAN LI \\ Department of Hematology, First Affiliation Hospital of China Medical University, Shenyang, Liaoning 110001, P.R. China
}

Received May 27, 2015; Accepted October 5, 2016

DOI: $10.3892 / 01.2016 .5289$

\begin{abstract}
Wnt signaling pathway, which regulates cell proliferation and differentiation. Activation of the $\mathrm{Wnt} / \beta$-catenin signaling pathway has been shown to positively correlate with prognosis in several types of malignancies. The present study aimed to determine the role of $\beta$-catenin in multiple myeloma (MM) cells using lentiviruses expressing small interfering RNA (siRNA). The expression of $\beta$-catenin in the MM cell line RPMI-8826 was evaluated following $\beta$-catenin knockdown by the siRNA. Subsequently, the activation of autophagy in MM cells was assessed by transmission electron microscopy and western blot analyses. Cell apoptosis and the expression of apoptosis-related proteins following $\beta$-catenin silencing was investigated by flow cytometry and western blotting, respectively. A significant decrease in $\beta$-catenin expression was observed in the MM cell line expressing $\beta$-catenin-specific siRNA. Activation of autophagy was induced by $\beta$-catenin silencing, as evidenced by increases in the number of autophagic vacuoles and the expression of the autophagy-related proteins microtubule-associated protein 1 light chain 3 and Beclin-1. Furthermore, the expression of 5'-adenosine monophosphate-activated protein kinase was increased, and that of mechanistic target of rapamycin was decreased, following $\beta$-catenin silencing. In addition, there was an increase in the rate of apoptosis of MM cells following $\beta$-catenin silencing, accompanied by increased protein expression of phosphorylated p53, active caspase-3 and B-cell lymphoma ( $\mathrm{Bcl})-2$-associated $\mathrm{X}$ protein, and decreased protein expression of $\mathrm{Bcl}-2$. The results of the present study suggested that $\beta$-catenin silencing induced autophagy and apoptosis in MM cells. To the best of our knowledge, this is the first study to demonstrate that a $\beta$-catenin deficiency induces autophagy in MM cells. These findings suggested that inhibition of $\beta$-catenin could be a potential therapeutic target for patients with MM.
\end{abstract}

Correspondence to: Dr Yan Li, Department of Hematology, First Affiliation Hospital of China Medical University, 155 Nanjing Road, Shenyang, Liaoning 110001, P.R. China

E-mail: beeperchild@163.com

Key words: multiple myeloma, Wnt/ $\beta$-catenin signaling, autophagy, apoptosis

\section{Introduction}

Multiple myeloma (MM) is a malignancy of plasma cells in the bone marrow. It is the second most common hematological cancer, accounting for $\sim 1 \%$ of all cancers and $>10 \%$ of all hematological neoplastic diseases $(1,2)$. As a result of its heterogeneous symptoms, the diagnosis of MM tends to be delayed, with an average median survival time of 3 to 4 years upon diagnosis. Newer therapeutic modalities such as autologous stem cell transplantation and the use of drugs, including thalidomide, lenalidomide and bortezomib, have considerably improved the clinical outcomes and survival of patients with MM (1,2). However, despite these advances in the treatment of MM, it remains an incurable disease owing to its protean clinical manifestations and complications.

MM is characterized by uncontrolled bone remodeling caused by the imbalance between bone resorption and bone formation resulting from increased osteoclastic activation with concomitant osteoblast suppression (3). Previous studies have demonstrated that the activation of the Wnt/ $\beta$-catenin signaling pathway has a critical role in both osteoblastogenesis and osteoclastogenesis $(4,5)$. In addition, aberrant activation of the Wnt/ $\beta$-catenin signaling pathway has been shown to be involved in numerous types of cancers $(6,7)$. In the absence of Wnt ligands, the downstream effector of the canonical Wnt signal, the transcription factor $\beta$-catenin, is phosphorylated by glycogen synthase kinase (GSK)-3 $\beta$. Phosphorylated $\beta$-catenin is then subjected to proteasome-mediated degradation to prevent its accumulation. Conversely, upon binding of Wnt ligands, the frizzled receptor and low-density lipoprotein receptor-related protein 5 or 6 (LRP5/6) form a complex and inhibit $\beta$-catenin phosphorylation. $\beta$-catenin is thus stabilized and translocated into the nucleus, where it associates with T-cell factor to permit DNA binding and the subsequent regulation of the expression of various target genes, including c-Myc and cyclin D1 (8-11). Activation of the Wnt/ $\beta$-catenin signaling pathway is frequently observed in MM and some other malignant tumors $(6,7)$. Since inhibition of Wnt/ $\beta$-catenin is known to suppress MM progression $(11,12)$, blockage of the Wnt/ $\beta$-catenin signaling pathway may prove to be a novel therapeutic approach.

Apoptosis and autophagy are two types of programmed cell death mechanisms implicated in cancer suppression $(13,14)$. Previous studies have shown that inhibition of the Wnt/ $\beta$-catenin signaling pathway attenuates survival 
signals and induces apoptosis $(15,16)$. Autophagy is a highly conserved pathway that is activated under stress and plays a pivotal role in a plethora of physiological processes including cell death (17). Previous studies have documented that autophagy negatively regulates Wnt signaling by promoting the degradation of dishevelled under metabolic stress $(18,19)$. Furthermore, $\beta$-catenin silencing induced both apoptotic and autophagic cell death in squamous cell carcinoma (20). However, the role of $\beta$-catenin signal in regulating autophagy has not been adequately researched.

RNA interference is a promising gene-silencing approach in the field of cancer therapeutics. In the present study, $\beta$-catenin silencing in MM cells was achieved by infection of the cells with a lentivirus vector encoding $\beta$-catenin-specific small interfering (si)RNA. The $\beta$-catenin-knockdown MM cells were then analyzed for any changes in the patterns of apoptosis or autophagy and the underlying mechanisms.

\section{Materials and methods}

Cell culture. The RPMI-8826 MM cell line was obtained from Fudan University Institutes of Biomedical Sciences cell bank, Shanghai, China. The cells were cultured in RPMI-1640 medium supplemented with $10 \%(\mathrm{v} / \mathrm{v})$ fetal bovine serum (Gibco; Thermo Fisher Scientific, Inc., Waltham, MA, USA) and $1 \%$ penicillin/streptomycin (Gibco; Thermo Fisher Scientific, Inc.) at $37^{\circ} \mathrm{C}$ and $5 \% \mathrm{CO}_{2}$ in a humidified incubator.

Development of a $\beta$-catenin-deficient $M M$ cell line. Three siRNA-targeting regions (siA, CCGGGCTTGGAAT GAGACTGCTGATCTCGAGATCAGCAGTCTCATTCCAAGC TTTTT; siB, CCGGCGCATGGAAGAAATAGTTGAACT CGAGTTCAACTATTTCTTCCATGCGTTTTT; siC, CCG GAGGTGCTATCTGTCTGCTCTACTCGAGTAGAGCAG ACAGATAGCACCTTTTTT) of the human CTNNB1 gene (encoding the $\beta$-catenin protein; GenBank accession no. NM_001,904) or scramble siRNA (sc, CCGGTTCTC CGAACGTGTCACGTTTCAAGAGAACGTGACACGTTC GGAGAATTTTTG) were designed, synthesized and ligated with the hU6MCS-Ubiquitin-EGFP-IRES-puromycin vector (Genechem, Shanghai, China). Lentivirus was packaged by transfection of recombinant constructs and package plasmids into $293 \mathrm{~T}$ cells (Genechem) using Lipofectamine 2000 (Genechem), and the viruses were then harvested after $48 \mathrm{~h}$ of transfection; the multiplicity of infection (MOI) for the virus was determined using the $293 \mathrm{~T}$ cells. To calculate the infection rate, images of 10 random fields of cells were captured under fluorescent channel and bright field, and the number of green fluorescent protein (GFP)-positive cells and total cells were counted, respectively. The infection rate was calculated by dividing the average number of GFP-positive cells by the average number of total cells.

To optimize the dose of puromycin required to kill RPMI-8826 cells, the cells were pretreated with different doses $(1,2.5,5$ or $10 \mu \mathrm{g} / \mathrm{ml})$ of puromycin (Genechem), after which the dose of $1 \mu \mathrm{g} / \mathrm{ml}$ puromycin was selected. To establish stable cells expressing $\beta$-catenin siRNA, RPMI- 8826 cells $\left(5 \times 10^{3} / \mathrm{ml}\right)$ were infected with lentiviruses expressing scramble or different $\beta$-catenin siRNAs at a MOI of 100, and the media was replaced after $8 \mathrm{~h}$ of infection. Puromycin at a concentration of $1 \mu \mathrm{g} / \mathrm{ml}$ was added after $72 \mathrm{~h}$ of infection, and maintained for 2 weeks. Cell counting kit (CCK)-8 (Dojindo Molecular Technologies, Inc., Shanghai, China) assays, western blotting and apoptosis assays were performed on RPMI-8826 myeloma cells stably expressing the different siRNAs.

CCK-8 assay. RPMI-8826 scramble (8826-sc) and $\beta$-catenin siRNA (8826-siC) cells ( $5 \times 10^{3}$ cells/well) $100 \mu$ l were seeded separately in 96-well plates, which had been pre-incubated for $24 \mathrm{~h}$ at $37^{\circ} \mathrm{C}$ in an atmosphere of $\mathrm{CO}_{2}$. A total of $10 \mu \mathrm{l} \mathrm{CCK}-8$ solution was added to each well and incubated for 1-4 h. The absorbance was then measured at a wavelength of $450 \mathrm{~nm}$ using a microplate reader (Thermo Fisher Scientific, Inc.).

Transmission electron microscope (TEM) analysis. A total of 1 million RPMI-8826 scramble (8826-sc) and $\beta$-catenin siRNA (8826-siC) expressing cells were centrifuged at $11,500 \mathrm{x}$ g for $5 \mathrm{~min}$ at $25^{\circ} \mathrm{C}$, and fixed with $4 \%$ glutaraldehyde for $2 \mathrm{~h}$ at room temperature. The cells were washed twice with PBS after fixing with osmic acid for $1 \mathrm{~h}$ at $4^{\circ} \mathrm{C}$. The samples were dehydrated and embedded with epoxy resin, after which 70-nm thick sections were cut. The sections were stained with uranyl acetate and lead citrate, and visualized under a TEM (H7650; Hitachi, Ltd., Tokyo, Japan) at x20,000 magnification.

Western blotting. The cells were washed thrice with PBS before being lysed with radioimmunoprecipitation assay buffer (Beijing Solarbio Science \& Technology Co., Ltd., Beijing, China). Protein concentrations were determined using the bicinchoninic acid protein assay kit (Beijing Solarbio Science \& Technology Co., Ltd.), according to the manufacturer's protocol. Equal amounts of protein lysate $(30 \mu \mathrm{g})$ were separated by $10 \%$ SDS-PAGE and transferred onto polyvinylidene difluoride membranes. After blocking with $5 \%$ skim milk, the membranes were incubated with primary antibodies $(1: 1,000)$ against $\beta$-catenin (cat. no. 9562), microtubule-associated protein 1 light chain 3 (LC3; cat. no. 3868), B-cell lymphoma (Bcl)-2 (cat. no. 2872), Bcl-2-associated X protein (Bax; cat. no. 2774), Beclin-1 (cat. no. 3495), phosphorylated-p53 (cat. no. 9284), phosphorylated-mechanistic target of rapamycin (mTOR; cat. no. 5536), phosphorylated-5'-adenosine monophosphate-activated protein kinase (AMPK; cat. no. 2535) and AMPK (cat. no. 2532) (all: Cell Signaling Technology, Inc., Danvers, MA, USA), active caspase-3 (cat. no. 1476-1; Epitomics, Burlingame, CA, USA) and $\beta$-actin (1:1,000; AA128, Beyotime, Shanghai, China) overnight at $4^{\circ} \mathrm{C}$. The same membrane was washed with Tris-buffered saline with Tween 20 and reblotted with peroxidase-conjugated affinipure goat anti-rabbit (cat. no. ZB-2301) and anti-mouse (cat. no. 2305) IgG secondary antibodies (1:10,000; ZSGB-BIO, Beijing, China) for $1 \mathrm{~h}$ at $25^{\circ} \mathrm{C}$. For quantitative analysis, the bands were selected and quantified using Image J 1.44 software (National Institutes of Health, Bethesda, MD, USA), and the data were transformed and normalized to $\beta$-actin.

Apoptosis assay by flow cytometry. The apoptosis of RPMI-8826 cells infected with lentiviruses encoding 


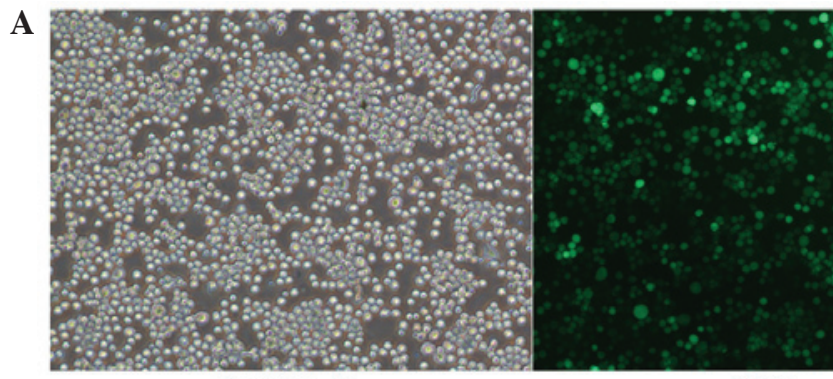

Bright field
GFP

$\mathbf{C}$
B

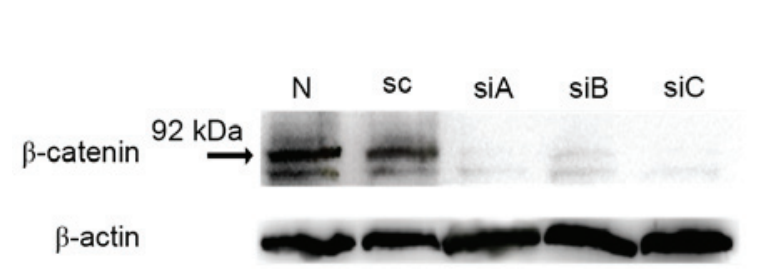

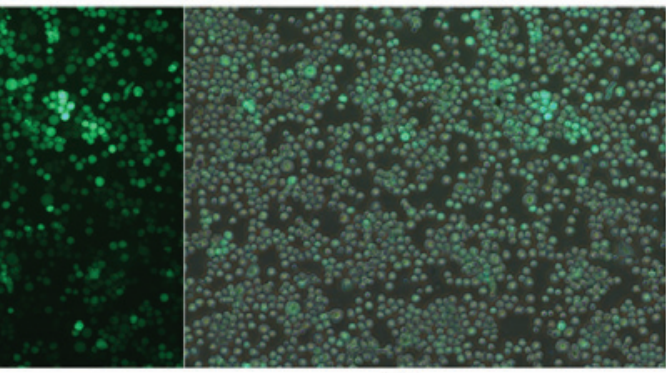

Merge

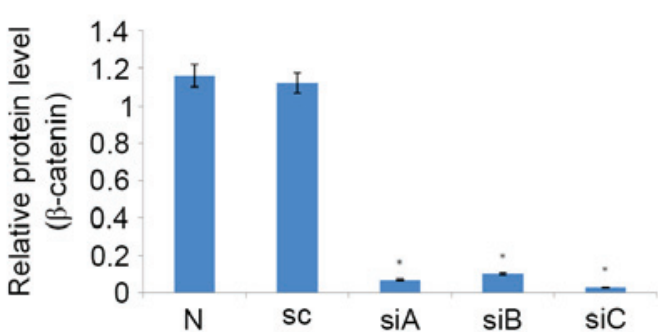

Figure 1. Knockdown efficacy of $\beta$-catenin by lentivirus-mediated siRNA. (A) Bright field, fluorescent and merged view of 8826 -siC cells at $72 \mathrm{~h}$ post-infection (magnification, $\mathrm{x} 200$ ). (B) Western blot showing effective silencing of $\beta$-catenin protein by lentivirus-mediated siRNA. Arrow indicates $\beta$-catenin band. (C) Quantification of western blot by densitometric analysis. "P<0.05 vs. SC group. N, untransfected RPMI-8826 cells; SC, RPMI-8826 cells infected with lentivirus carrying scramble siRNA; siA-C, RPMI-8826 cells infected with lentiviruses carrying $\beta$-catenin-specific siRNAs; siRNA, small interfering RNA; GFP, green fluorescent protein.

scramble or $\beta$-catenin-specific siRNAs was assessed by flow cytometry using the Annexin V/Propidium iodide-fluorescein isothiocyanate Apoptosis Detection kit (BD Biosciences, Franklin Lakes, NJ, USA), according to the manufacturer's protocol. Cells treated with PBS were used as negative controls.

Statistical analysis. Data are expressed as the mean \pm standard deviation. Student's t-tests were performed using SPSS 11.0 software (SPSS, Inc., Chicago, IL, USA). P<0.05 was considered statistically significant.

\section{Results}

Transfection efficacy of $\beta$-catenin siRNA lentivirus in a MM cell line. To evaluate the effect of $\beta$-catenin silencing in $\mathrm{MM}$ cells, siRNAs were designed by targeting different regions of $\beta$-catenin. RPMI-8826 MM cells were infected with lentiviruses expressing scramble siRNA (8826-sc) or three $\beta$-catenin siRNAs (8826-siA, siB and siC), and selected with puromycin. Non-transfected cells were used as a control. As shown in Fig. 1A, $70 \%$ of cells were GFP-positive after $72 \mathrm{~h}$ of infection, whereas no GFP-positive cells were identified in the control group (data not shown), suggesting that RPMI-8826 MM cells were successfully infected with the lentiviruses expressing siRNAs. In addition, to further validate the targeting of $\beta$-catenin by siRNA, stable cells expressing $\beta$-catenin siRNA were harvested for western blot analysis. As shown in Fig. 1B, non-transfected and scramble siRNA (8826-sc) transfected cells expressed high levels of $\beta$-catenin protein, which is consistent with a previous study (21). Furthermore, all siRNAs had a >90\% knockdown efficacy, as compared with the scramble siRNA (8826-sc)

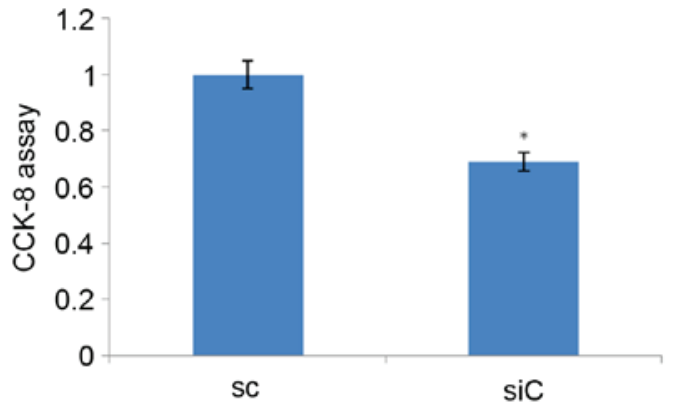

Figure 2. CCK- 8 assay to evaluate cell viability following silencing of $\beta$-catenin in multiple myeloma cells. ${ }^{*} \mathrm{P}<0.05$ vs. SC. CCK- 8 , cell counting kit-8; siC, RPMI-8826 cells infected with lentivirus carrying $\beta$-catenin-specific small interfering RNA.

$(\mathrm{P}<0.05$; Fig. 1B and $\mathrm{C})$. However, siC showed slightly more effective silencing than siA and $\mathrm{siB}$, and the difference was significant $(\mathrm{P}=0.017$ for siA vs. $\mathrm{siC} ; \mathrm{P}=0.003$ for $\mathrm{siB}$ vs. siC; Fig. 1C). Therefore, siC was selected for the subsequent analyses. The slightly different knockdown efficacies among these three siRNAs may have occurred as a result of different target regions and infection rates.

$\beta$-catenin silencing decreases the viability of MM cells. CCK- 8 assays were performed to evaluate the anti-proliferative effect of $\beta$-catenin silencing on MM cells. Cell viabilities of 8826-sc and 8826-siC cells were monitored. As shown in Fig. 2, the cell viability was decreased by $16.4 \%$ in $8826-$ siC cells compared with 8826-sc cells, and the difference was significant $(\mathrm{P}=0.019)$.

Autophagy is induced in $\beta$-catenin-deficient MM cells. After confirming the successful silencing of $\beta$-catenin in MM cells 
A

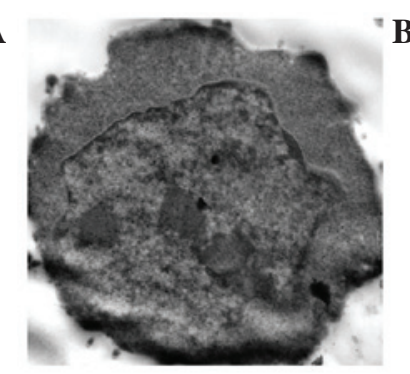

D

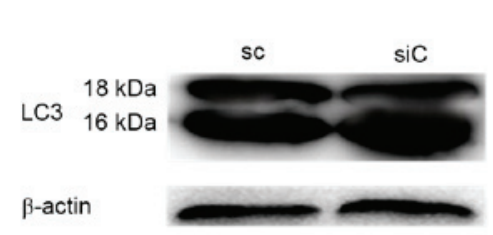

$\mathbf{F}$

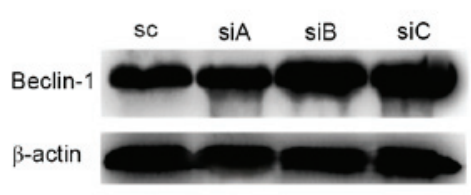

$\mathrm{siC}$

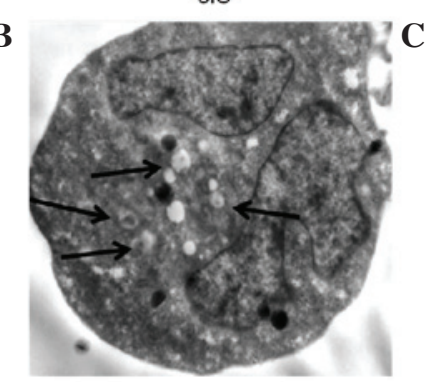

$\mathbf{E}$

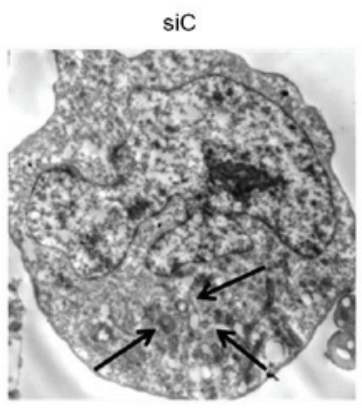

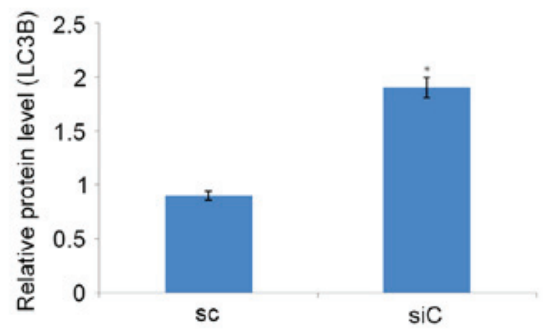

G

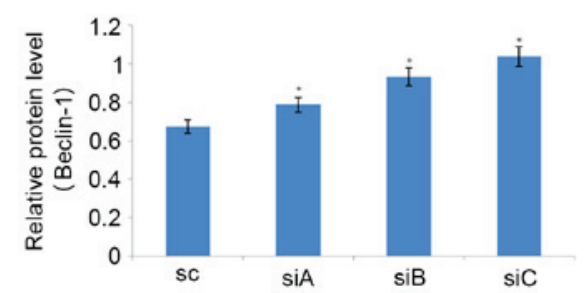

Figure 3. Silencing of $\beta$-catenin induces autophagy. (A-C) Transmission electron microscopy analysis of RPMI-8226 cells following $\beta$-catenin knockdown by siRNA. Arrow indicates autophagic vacuoles (magnification, $\mathrm{x} 15,000$ ). (D and E) Western blot analysis demonstrated that the protein expression levels of LC3 were significantly increased in RPMI-8826 cells following $\beta$-catenin silencing. (F and G) Western blot analysis demonstrated that the protein expression levels of Beclin-1 were significantly increased in RPMI- 8826 cells following $\beta$-catenin silencing. ${ }^{*} \mathrm{P}<0.05$ vs. SC. SC, RPMI- 8826 cells infected with lentivirus carrying scramble siRNA; siA-C, RPMI-8826 cells infected with lentiviruses carrying $\beta$-catenin-specific siRNAs; siRNA, small interfering RNA; LC3, microtubule-associated protein 1 light chain 3.

A

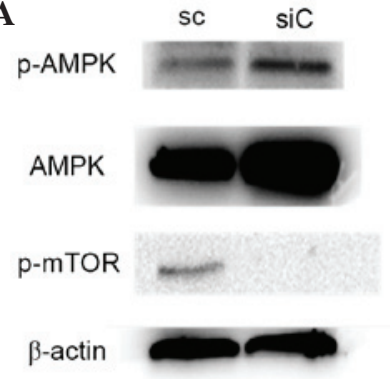

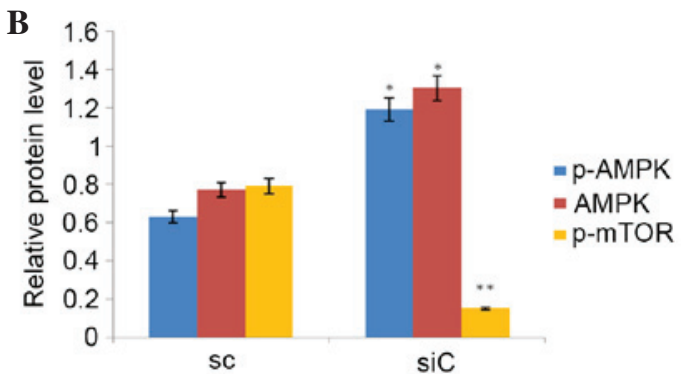

Figure 4. Regulation of the AMPK/mTOR pathway by $\beta$-catenin silencing. (A) Western blot analysis demonstrated that the protein expression levels of p-AMPK and total AMPK were increased following $\beta$-catenin knockdown in multiple myeloma cells, while $\mathrm{p}$-mTOR was reduced by $\beta$-catenin silencing. (B) Quantification of the western blot using ImageJ software. " $\mathrm{P}<0.05$ and ${ }^{* * *} \mathrm{P}<0.01$ vs. SC. p-AMPK, phosphorylated-5'-adenosine monophosphate-activated protein kinase; p-mTOR, phosphorylated-mechanistic target of rapamycin; SC, RPMI-8826 cells infected with lentivirus carrying scramble siRNA; siC, RPMI-8826 cells infected with lentivirus carrying $\beta$-catenin-specific siRNA; siRNA, small interfering RNA.

using siRNA, the present study determined whether autophagy was induced as a result of $\beta$-catenin silencing. Upon TEM analysis to assess cytoplasmic changes in 8826-siC and 8826-sc cells, autophagic vacuoles (round enclosed compartments) were observed in the former but not in the latter cells (Fig. 3A-C). To further confirm $\beta$-catenin-deficiency-induced autophagy, the protein expression levels of LC3 and Beclin-1, which are indicators of autophagosome formation and activation of the autophagy pathway (22), were examined. $\beta$-catenin silencing induced LC3-I (18 kDa) cleavage into LC3-II (16 kDa), resulting in increased LC3-II and decreased LC-I expression (Fig. 3D and E). $\beta$-catenin silencing also significantly increased the levels of Beclin-1 protein $(\mathrm{P}<0.05$; Fig. $3 \mathrm{~F}$ and $\mathrm{G})$. These results suggest that $\beta$-catenin silencing in myeloma cell lines induces autophagy. 
A

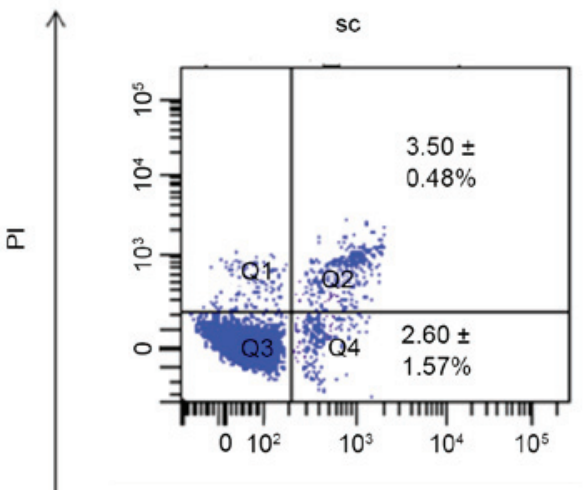

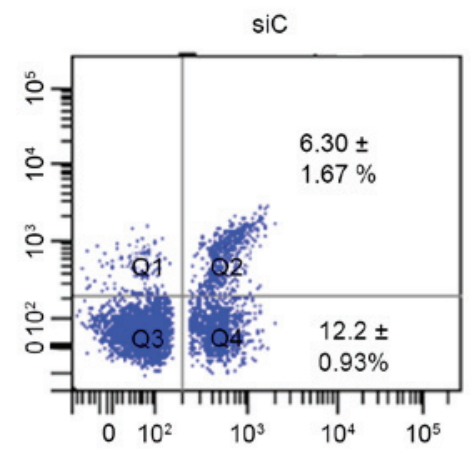

Annexin V

B
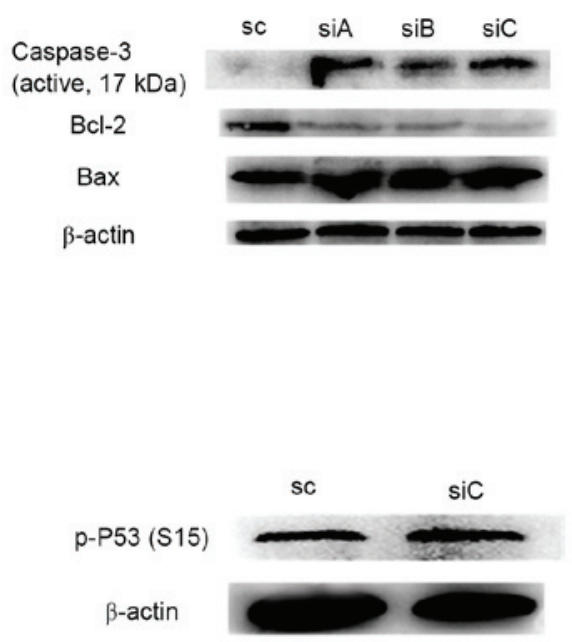
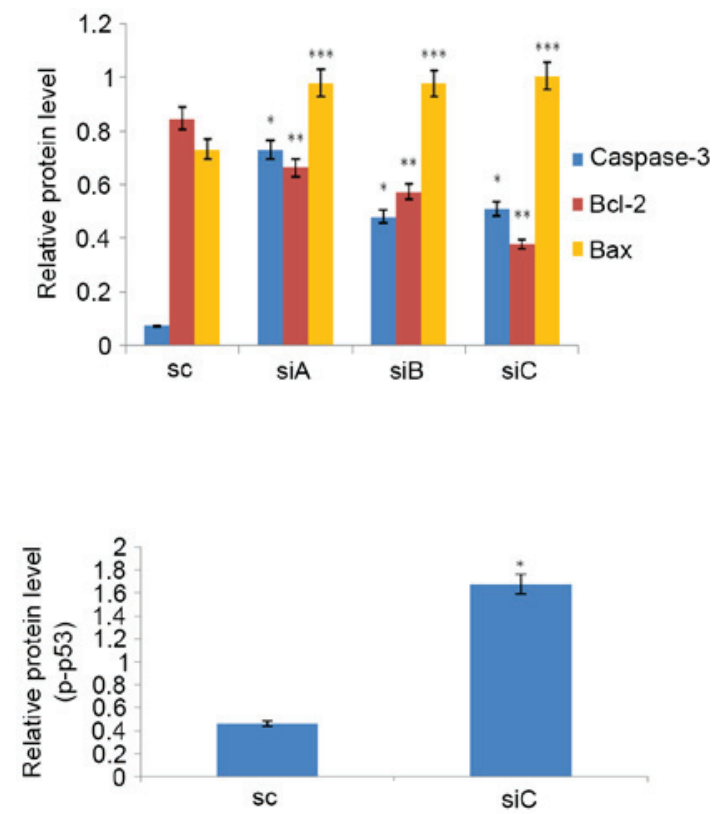

Figure 5. Activation of apoptosis following silencing of $\beta$-catenin. (A) Annexin V and PI staining followed by flow cytometry demonstrated increased apoptosis of MM cells following $\beta$-catenin knockdown. (B) Western blotting demonstrated that the protein expression levels of p-p53, active caspase-3 and Bax were increased, and those of $\mathrm{Bcl}-2$ were decreased, in $\mathrm{MM}$ cells following $\beta$-catenin knockdown. ${ }^{*} \mathrm{P}<0.001,{ }^{* *} \mathrm{P}<0.01$ and ${ }^{* * * *} \mathrm{P}<0.05$ vs. SC. MM, multiple myeloma; PI, propidium iodide; p-p53, phosphorylated-p53; Bcl-2, B-cell lymphoma-2; Bax, B-cell lymphoma-2-associated X protein; SC, RPMI-8826 cells infected with lentivirus carrying scramble siRNA; siA-C, RPMI-8826 cells infected with lentiviruses carrying $\beta$-catenin-specific siRNAs; siRNA, small interfering RNA.

Involvement of the AMPK/mTOR pathway in $\beta$-catenin knockdown-induced autophagy. The present study investigated whether the AMPK/mTOR signaling pathway is involved in the activation of autophagy by $\beta$-catenin silencing. In 8826-siC cells, the expression levels of both phosphorylated-AMPK and total AMPK were significantly increased, as compared with 8826-sc cells $(\mathrm{P}<0.05$; Fig. 4A and B), while the expression levels of phosphorylated-mTOR were significantly decreased in 8826-siC cells compared with 8826-sc cells $(\mathrm{P}<0.05$; Fig. 4A and B). These results suggest that the AMPK/mTOR pathway is activated by $\beta$-catenin silencing and may be involved in the activation of autophagy.

$\beta$-catenin silencing increases the apoptosis of RPMI-8826 cells. The present study determined whether apoptosis was induced by knockdown of $\beta$-catenin in a MM cell line. To determine the activation of apoptosis, the Annexin V/propidium iodide (PI) assay was performed to analyze 8826-siC and 8826 -sc cells by flow cytometry. As shown in Fig. 5A, $12.2 \pm 0.93$ and $6.30 \pm 1.67 \%$ of the $8826-$ siC cells were Annexin $\mathrm{V}^{+} / \mathrm{PI}^{-}$and Annexin $\mathrm{V}^{+} / \mathrm{PI}^{+}$, respectively, which was markedly higher than 8826 -sc cells $(2.60 \pm 1.57 \%$ Annexin $\mathrm{V}^{+} / \mathrm{PI}^{-}$and $3.50 \pm 0.48 \%$ Annexin $\mathrm{V}^{+} / \mathrm{PI}^{+}$), suggesting that apoptosis was induced by $\beta$-catenin silencing in myeloma cells. Subsequently, the protein expression of apoptosis-related proteins, including p53, active caspase-3, Bax and Bcl-2, was evaluated. As shown in Fig. 4B, $\beta$-catenin silencing resulted in increased protein expression of phosphorylated-p53, active caspase-3 and Bax, and decreased protein expression of Bcl-2 ( $\mathrm{P}<0.001$; Fig. 5B). Together, these findings suggest that $\beta$-catenin silencing promoted apoptosis in MM cells probably via the mitochondrial apoptotic pathway. 


\section{Discussion}

MM is an incurable disease characterized by malignant proliferation of plasma cells in the bone marrow. The canonical Wnt/ $\beta$-catenin signaling pathway, which is related to MM cell growth, survival and migration, is highly active in MM cells $(4,11)$. The present study aimed to investigate the role of $\beta$-catenin in apoptosis and autophagy in MM cells by silencing $\beta$-catenin using a lentivirus vector carrying $\beta$-catenin-specific siRNA. The results of the present study demonstrated that both apoptosis and autophagy were induced by $\beta$-catenin silencing, suggesting its value as a potential therapeutic target in the treatment of MM.

The canonical Wnt/ $\beta$-catenin signaling pathway is recurrently aberrant in several types of cancer, including MM (22). Binding of Wnt ligands to frizzed and LRP5/6 receptors induces the suppression of GSK-3 $\beta$ activity and accumulation of $\beta$-catenin in the nucleus, leading to activation of downstream target genes such as c-Myc and cyclin D1 (8-11). A previous study demonstrated $\beta$-catenin expression in nearly all primary MM cells, whereas it was absent in normal plasma cells (23). Several studies have shown that treatment with Wnt ligand or GSK-3 $\beta$ inhibitor significantly increased the accumulation of $\beta$-catenin and MM cell proliferation, indicating highly activated $\mathrm{Wnt} / \beta$-catenin signaling in $\mathrm{MM}$ cells $(23,24)$. In the present study, the effect of $\beta$-catenin knockdown in human RPMI-8826 MM cells was examined. High expression of $\beta$-catenin was detected in the MM cells prior to silencing, which was consistent with a previous study (21). However, significantly decreased protein expression levels of $\beta$-catenin were observed following $\beta$-catenin silencing, suggesting that $\beta$-catenin silencing may present a promising strategy for cancer therapy.

Autophagy is a lysosomal degradation pathway that has a critical role in cell survival and differentiation. It serves as an adaptive mechanism to protect organisms in response to stresses or pathological states such as infection and cancer (25). Previous studies have demonstrated that the mTOR inhibitor rapamycin and nutrient deficiencies attenuate Wnt signaling, while knockdown of the autophagy effectors, LC3 or Beclin-1, increases Wnt-induced transcriptional activity, suggesting that Wnt signaling downregulates autophagy $(18,26)$. These findings suggest an important role for the Wnt/ $\beta$-catenin signaling pathway in the process of autophagy. To the best of our knowledge, this is the first study to report an association between Wnt/ $\beta$-catenin signaling and autophagy regulation in MM cells. In the present study, $\beta$-catenin induced autophagy in MM cells, as evidenced by the presence of autophagic vacuoles and increased expression of LC 3 and Beclin-1. In addition, the expression of mTOR, a key negative regulator of autophagy (27), was decreased. Autophagy is promoted by AMPK, which is a key energy sensor and regulates cellular metabolism to maintain energy homeostasis (28). The present study demonstrated that AMPK was activated by $\beta$-catenin silencing, indicating its involvement in the activation of autophagy.

In addition to autophagy, a pro-apoptotic effect of silencing $\beta$-catenin in MM cells was observed. $\beta$-catenin silencing increased the protein expression of phosphorylated-p53, the pro-apoptotic protein Bax and active caspase-3, while it decreased the expression of the anti-apoptotic protein, Bcl-2.
These findings suggested that $\beta$-catenin silencing promoted apoptosis in MM cells, potentially via activation of the mitochondrial apoptotic pathway. The role of $\beta$-catenin in the regulation of cell proliferation and apoptosis differs in different cell types. For example, $\beta$-catenin accumulation induces the proliferation and survival of some tumor cells $(29,30)$, which is similar to the findings of the present study. Conversely, in hematopoietic progenitor cells, activation of $\beta$-catenin induces apoptosis via the mitochondrial pathway (31). There is a complex relationship between autophagy and apoptosis, which may vary in different biological contexts (25). Both can share overlapping functions, or have modifying effects on each other (25). In the present study, parallel activation of autophagy and apoptosis by $\beta$-catenin silencing in MM cells, both of which are related to cell death, was observed.

In conclusion, the present study demonstrated that $\beta$-catenin silencing induced autophagy as well as apoptosis in MM cells. Therefore, inhibition of $\beta$-catenin may be considered a promising strategy for the treatment of MM. Further studies are required to further delineate the specific pathways underlying the activation of autophagy and apoptosis in $\beta$-catenin-knockdown MM cells.

\section{Acknowledgements}

This study was supported by Liaoning Bureau of Science and Technology Science and Technology program (grant no. 2013021031).

\section{References}

1. Palumbo A and Anderson K: Multiple myeloma. N Engl J Med 364: 1046-1060, 2011.

2. Hideshima T, Mitsiades C, Tonon G, Richardson PG and Anderson KC: Understanding multiple myeloma pathogenesis in the bone marrow to identify new therapeutic targets. Nat Rev Cancer 7: 585-598, 2007.

3. Roodman GD: Pathogenesis of myeloma bone disease. Leukemia 23: 435-441, 2009.

4. Qiang YW, Chen Y, Brown N, Hu B, Epstein J, Barlogie B and Shaughnessy JD Jr: Characterization of Wnt/beta-catenin signalling in osteoclasts in multiple myeloma. Br J Haematol 148: 726-738, 2010.

5. Houben A, Kostanova-Poliakova D, Weissenböck M, Graf J, Teufel S, von der Mark K and Hartmann C: $\beta$-catenin activity in late hypertrophic chondrocytes locally orchestrates osteoblastogenesis and osteoclastogenesis. Development: Sep 12, 2016 (Epub ahead of print).

6. Morin PJ: Beta-catenin signaling and cancer. Bioessays 21: 10211030,1999

7. Zhan T, Rindtorff $\mathrm{N}$ and Boutros $\mathrm{M}$ : Wnt signaling in cancer. Oncogene: Sep 12, 2016 (Epub ahead of print).

8. Zhang B, Abreu JG, Zhou K, Chen Y, Hu Y, Zhou T, He X and Ma JX: Blocking the Wnt pathway, a unifying mechanism for an angiogenic inhibitor in the serine proteinase inhibitor family. Proc Natl Acad Sci USA 107: 6900-6905, 2010.

9. Macdonald BT, Semenov MV and He X: SnapShot: Wnt/beta-catenin signaling. Cell 131: 1204, 2007.

10. Qi W, Yang C, Dai Z, Che D, Feng J, Mao Y, Cheng R, Wang Z, He X, Zhou T, et al: High levels of pigment epithelium-derived factor in diabetes impair wound healing through suppression of wnt signaling. Diabetes 64: 1407-1419, 2015.

11. Sukhdeo K, Mani M, Zhang Y, Dutta J, Yasui H, Rooney MD, Carrasco DE, Zheng M, He H, Tai YT, et al: Targeting the beta-catenin/TCF transcriptional complex in the treatment of multiple myeloma. Proc Natl Acad Sci USA 104: 7516-7521, 2007.

12. Fulciniti M, Tassone $P$, Hideshima T, Vallet $S$, Nanjappa $P$, Ettenberg SA, Shen Z, Patel N, Tai YT, Chauhan D, et al: Anti-DKK1 mAb (BHQ880) as a potential therapeutic Agent for multiple myeloma. Blood 114: 371-379, 2009. 
13. Tsujimoto Y and Shimizu S: Another way to die: Autophagic programmed cell death. Cell Death Differ 12 (Supp 2): S1528-S1534, 2005.

14. Bursch W, Ellinger A, Gerner C, Frohwein U and Schulte-Hermann R: Programmed cell death (PCD). apoptosis, autophagic PCD, or others? Ann N Y Acad Sci 926: $1-12,2000$.

15. Chen S, Guttridge DC, You Z, Zhang Z, Fribley A, Mayo MW, Kitajewski J and Wang CY: Wnt-1 signaling inhibits apoptosis by activating beta-catenin/T cell factor-mediated transcription. J Cell Biol 152: 87-96, 2001.

16. Hseu YC, Thiyagarajan V, Tsou HT, Lin KY, Chen HJ, Lin CM, Liao JWand Yang HL: In vitro and in vivo anti-tumor activity of CoQ0 against melanoma cells: inhibition of metastasis and induction of cell-cycle arrest and apoptosis through modulation of Wnt/ $\beta$-catenin signaling pathways. Oncotarget 7: 22409-22426, 2016.

17. Puissant A, Robert G and Auberger P: Targeting autophagy to fight hematopoietic malignancies. Cell Cycle 9: 3470-3478, 2010

18. Gao C, Cao W, Bao L, Zuo W, Xie G, Cai T, Fu W, Zhang J, Wu W, Zhang $\mathrm{X}$ and Chen YG: Autophagy negatively regulates Wnt signalling by promoting dishevelled degradation. Nat Cell Biol 12: 781-790, 2010.

19. He C and Klionsky DJ: Regulation mechanisms and signaling pathways of autophagy. Annu Rev Genet 43: 67-93, 2009

20. Chang HW, Lee YS, Nam HY, Han MW, Kim HJ, Moon SY, Jeon H, Park JJ, Carey TE, Chang SE, et al: Knockdown of beta-catenin controls both apoptotic and autophagic cell death through LKB1/AMPK signaling in head and neck squamous cell carcinoma cell lines. Cell Signal 25: 839-847, 2013.

21. Ashihara E, Kawata E, Nakagawa Y, Shimazaski C, Kuroda J, Taniguchi K, Uchiyama H, Tanaka R, Yokota A, Takeuchi M, et al: Beta-catenin small interfering RNA successfully suppressed progression of multiple myeloma in a mouse model. Clin Cancer Res 15: 2731-2738, 2009.

22. Moon RT, Kohn AD, De Ferrari GV and Kaykas A: WNT and beta-catenin signalling: diseases and therapies. Nat Rev Genet 5: 691-701, 2004.
23. Derksen PW, Tjin E, Meijer HP, Klok MD, MacGillavry HD, van Oers MH, Lokhorst HM, Bloem AC, Clevers H, Nusse R, et al: Illegitimate WNT signaling promotes proliferation of multiple myeloma cells. Proc Natl Acad Sci USA 101: 6122-6127, 2004.

24. Takada K, Zhu D, Bird GH, Sukhdeo K, Zhao JJ, Mani M, Lemieux M, Carrasco DE, Ryan J, Horst D, et al: Targeted disruption of the BCL9/ $\beta$-catenin complex inhibits oncogenic Wnt signaling. Sci Trans1 Med 4: 148ra117, 2012.

25. Levine B and Kroemer G: Autophagy in the pathogenesis of disease. Cell 132: 27-42, 2008.

26. Sukhdeo K, Mani M, Hideshima T, Takada K, Pena-Cruz V, Mendez G, Ito S, Anderson KC and Carrasco DR: Beta-catenin is dynamically stored and cleared in multiple myeloma by the proteasome-aggresome-autophagosome-lysosome pathway. Leukemia 26: 1116-1119, 2012.

27. Jung $\mathrm{CH}$, Ro SH, Cao J, Otto NM and Kim DH: mTOR regulation of autophagy. FEBS Lett 584: 1287-1295, 2010.

28. Kim J, Kundu M, Viollet B and Guan KL: AMPK and mTOR regulate autophagy through direct phosphorylation of Ulk1. Nat Cell Biol 13: 132-141, 2011.

29. Tetsu O and McCormick F: Beta-catenin regulates expression of cyclin D1 in colon carcinoma cells. Nature 398: 422-426, 1999.

30. He TC, Sparks AB, Rago C, Hermeking H, Zawel L, da Costa LT, Morin PJ, Vogelstein B and Kinzler KW: Identification of c-MYC as a target of the APC pathway. Science 281: 1509-1512, 1998.

31. Ming M, Wang S, Wu W, Senyuk V, Le Beau MM, Nucifora G and Qian Z: Activation of Wnt/beta-catenin protein signaling induces mitochondria-mediated apoptosis in hematopoietic progenitor cells. J Biol Chem 287: 22683-22690, 2012. 